\title{
Same sign WW scattering process as a probe of Higgs boson in pp collision at $\sqrt{s}=10 \mathrm{TeV}$
}

\author{
Bo Zhu ${ }^{1, a}$, Pietro Govoni ${ }^{2,3,4}$, Yajun $\mathbf{M a o}^{1}$, Chiara Mariotti ${ }^{5}$, Weimin $\mathbf{W u}^{6}$ \\ ${ }^{1}$ School of Physics, and State Key Laboratory of Nuclear Physics \& Technology, Peking University, Beijing, China \\ ${ }^{2}$ Milano-Bicocca University, Milano, Italy \\ ${ }^{3}$ INFN Milano-Bicocca, Milano, Italy \\ ${ }^{4}$ European Organization for Nuclear Research, Geneva, Switzerland \\ ${ }^{5}$ INFN Torino, Torino, Italy \\ ${ }^{6}$ Fermi National Accelerator Laboratory, Batavia, IL, USA
}

Received: 19 October 2010 / Revised: 25 November 2010 / Published online: 8 January 2011

(C) The Author(s) 2010. This article is published with open access at Springerlink.com

\begin{abstract}
WW scattering is an important process to study electroweak symmetry breaking in the Standard Model at the LHC, in which the Higgs mechanism or other new physics processes must intervene to preserve the unitarity of the process below $1 \mathrm{TeV}$. This channel is expected to be one of the most sensitive to determine whether the Higgs boson exists. In this paper, the final state with two same sign Ws is studied, with a simulated sample corresponding to the integrated luminosity of $60 \mathrm{fb}^{-1}$ in pp collision at $\sqrt{s}=10 \mathrm{TeV}$. Two observables, the invariant mass of $\mu \mu$ from $\mathrm{W}$ decays and the azimuthal angle difference between the two $\mu \mathrm{s}$, are utilized to distinguish the Higgs boson existence scenario from the Higgs boson absence scenario. A good signal significance for the two cases can be achieved. If we define the separation power of the analysis as the distance, in the loglikelihood plane, of pseudo-experiments outcomes in the two cases, with the total statistics expected from the ATLAS and CMS experiments at the nominal center-of-mass energy of $14 \mathrm{TeV}$, the separation power will be at the level of $4 \sigma$.
\end{abstract}

\section{Introduction}

It is predicted by the Standard Model (SM) that perturbative unitarity is violated in vector boson scattering process at high energy if the Higgs particle is absent [1-6]. This implies that the existence of a Higgs boson or new physics must intervene below $1 \mathrm{TeV}$. If the Higgs boson does exist, a resonance could be observed in the VV (WW or ZZ) invariant

a e-mail: zhub@pku.edu.cn

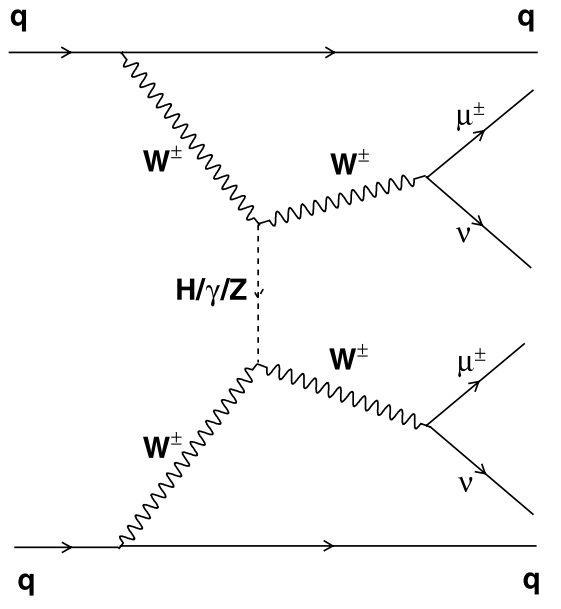

Fig. 1 Same sign WW scattering diagram

mass spectrum. On the other hand, new physics may appear in the form of vector boson pair resonances, as predicted by Little Higgs, Dynamical symmetry breaking, or Higgsless models [1-6]. Therefore, a measurement of WW scattering processes is a model independent approach to probe the existence or absence of a Higgs boson.

The same sign $\mathrm{WW}$ scattering with $\mathrm{W}$ decaying to $\mu \nu$ is expected to be a very clean process to study the difference between the standard model and new physics scenarios [7]. It has the best separation power between the two scenarios with respect to the other final states (WW, ZZ, WZ) as shown in [8]. It will help clarify the electroweak breaking mechanism in case a Higgs boson like resonance will not be observed or to finally test the unitarity of the theory. A characteristic signature of the same sign WW scattering is the presence of two forward jets (tag jets) with high energy (see Fig. 1) which can thus be efficiently extracted from 


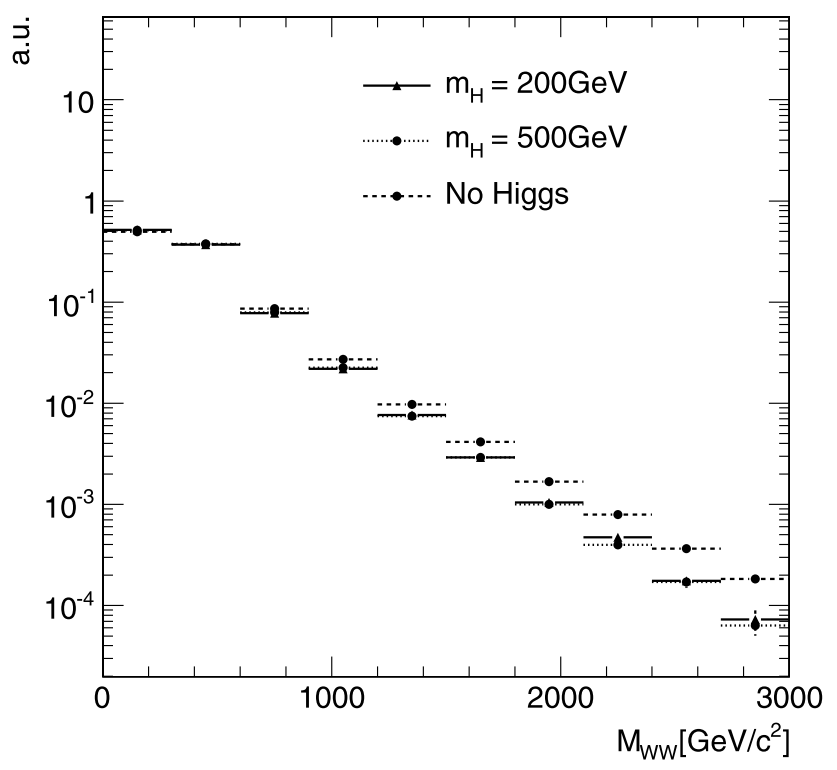

Fig. $2 m_{\mathrm{WW}}$ distribution for $m_{\mathrm{H}}=200 \mathrm{GeV} / \mathrm{c}^{2}, m_{\mathrm{H}}=500 \mathrm{GeV} / \mathrm{c}^{2}$ and no-Higgs Scenarios. The distribution is normalized to 1

most backgrounds. The other signature, namely the presence of a same sign isolated muons pair, can help in suppressing other backgrounds. In this work, we take into account all the possible backgrounds, including that due to the misidentification of leptons (which is usually neglected in other same sign WW scattering studies). We will show that we can get an almost background free result with the help of isolation techniques. The final state with 2 electrons or 1 electron and 1 muons have been studied, but the background subtraction result is much less effective, due to the high rate of mis-identified electrons.

Two same sign WW are produced only via t-channel process, thus no resonances are expected in the $m_{\mathrm{WW}}$ spectrum. The invariant mass of the WW is shown in Fig. 2 at parton level for two different values of the Higgs boson mass and for the case of no-Higgs. Because of the Parton Distribution Functions, the expected rise at large $m_{\mathrm{WW}}$ values is dramatically suppressed, but still a substantial difference between the two scenarios (Higgs and "no-Higgs") can clearly be observed.

\section{Monte Carlo samples}

The PHANTOM events generator [9] is used to generate $q q \rightarrow q q \mu^{ \pm} v \mu^{ \pm} v$ processes at $\mathcal{O}\left(\alpha_{\mathrm{EW}}^{6}\right)$, since it performs the full calculations at $\mathcal{O}\left(\alpha_{\mathrm{EW}}^{6}+\alpha_{\mathrm{EW}}^{4} \alpha_{\mathrm{S}}^{2}\right)$ order. This is necessary, since the study aims at comparing the WW scattering spectra under two different Higgs boson hypotheses: thus it is of crucial importance to correctly calculate the cross sections, by considering the interferences between the various tree-level diagrams present in the WW scattering process calculation.

Different Higgs boson hypotheses samples are generated for the signal: $m_{\mathrm{H}}=200 \mathrm{GeV} / \mathrm{c}^{2}, m_{\mathrm{H}}=500 \mathrm{GeV} / \mathrm{c}^{2}$ and no-Higgs scenarios. Out of all the possible diagrams calculated by PHANTOM, the WW scattering process is isolated by means of the following cuts at parton level: the invariant mass constraint $\left|m_{\mu v}-m_{\mathrm{W}}\right|<10 \mathrm{GeV} / \mathrm{c}^{2}$, the pseudo-rapidity difference of the final state quarks $\Delta \eta_{q q}>$ 2.0 , the invariant mass of the quarks $m_{q q}>300 \mathrm{GeV} / \mathrm{c}^{2}$, the minimal angle between the final state muon and quark $\Delta R(\mu q)^{\min }>1.2$. After these selections surviving events are considered as signal events, the remaining events are studied as irreducible background.

Besides the irreducible background, some other processes at $\mathcal{O}\left(\alpha_{\mathrm{EW}}^{4} \alpha_{s}^{2}\right)$ [9] with the same final states particles are also produced by PHANTOM. These processes are denoted as "QCD background" in the following.

The $t \bar{t} \rightarrow \mathrm{W}^{+} \mathrm{bW}^{-} \mathrm{b}$ production is another very important background, in which one hard muon comes from $\mathrm{W}$, the other same sign muon is from a b-hadron leptonic decay. Single top quark in association with $\mathrm{W}$ process is also considered because of the same reason.

The production of single $\mathrm{W}$ along with jets, in which the $\mathrm{W}$ decays into $\mu \nu$ is another dangerous background, because charged long lived hadrons $\left(k^{ \pm}, \pi^{ \pm}, p^{ \pm}\right)$may be wrongly identified as muons, and the large cross section compensates for the low probability of the mis-identification. We assume the probability of mis-identification to be $5 \times 10^{-4}$ [10]. In addition to the dominant backgrounds discussed above, single top, $t \bar{t} \mathrm{~W}$ and di-boson backgrounds (WW, WZ and ZZ) are studied as well.

QCD and irreducible background samples are produced with PHANTOM, $t \bar{t}, \mathrm{~W}+$ jet and $t \bar{t} \mathrm{~W}$ backgrounds are generated with Madgraph [11, 12] and the other backgrounds are simulated with PYTHIA [13] at a collision energy of $\sqrt{s}=10 \mathrm{TeV}$. The cross sections of the samples which are produced by PHANTOM are calculated at the Leading Order (LO), the cross sections of the other samples are calculated at the Next-to-Leading Order (NLO). The cross section will be roughly doubled if the collision energy is raised from 10 to $14 \mathrm{TeV}$. In all cases including signal and background samples, the parton showering and hadronization are performed with PYTHIA, and the jet reconstruction algorithm is also provided by PYTHIA. To include the detector effect, the muons and jets momenta are smeared by a gaussian distribution with the resolution based on the following $p_{T}$ resolution parameterization [14], for muons:

$$
\frac{\sigma\left(p_{T}\right)}{p_{T}}=e^{-4+0.0014 \times p_{T}}
$$


for jets:

$$
\frac{\sigma\left(p_{T}\right)}{p_{T}}=\sqrt{\frac{0.813^{2}}{p_{T}}+\frac{3.9^{2}}{p_{T}^{2}}+0.017^{2}} .
$$

\section{Event selection}

The aim of the selection strategy is to achieve a reasonable level of signal over background ratio. We concentrate on a cut-based selection strategy. The selection chain includes two main parts: muon selection and jet selection.

A pair of same sign isolated hard muons is one of the most significant characteristics of the signal process. Most standard model background events, such as $\mathrm{W}+$ jet, $t \bar{t}$, single top and di-boson, comprise only one muon or two opposite charged muons in the final state. If there are two same sign muons in these events, one muon should come from b-hadron decay or muon mis-identification from other backgrounds. Most of the non-top background events contain at least one fake muon mostly in the low $p_{T}$ region. A $p_{T}$ threshold of $15 \mathrm{GeV} / \mathrm{c}$ is required to suppress these kinds of background, especially the $\mathrm{W}+$ jet events.

The muon isolation criteria are applied to all the tracks of charged particles, which can be well reconstructed with an efficiency of almost $100 \%$ when $p_{T}>0.5 \mathrm{GeV} / \mathrm{c}$ [14]. The isolation parameter is defined as the sum of the $p_{T}$ of charged particles in an isolation cone of $0.3 \mathrm{rad}$ centered around the muon at the primary vertex, in the $(\eta, \phi)$ plane. The footprint of the muon itself is removed by an inner veto cone of $0.01 \mathrm{rad}$ :

$\beta=\Sigma p_{T}(0.01<\Delta R<0.3)$.

As the top background is the most important one, the following isolation cuts are tuned to reduce this contribution: $\beta<1 \mathrm{GeV} / \mathrm{c}$ and $\beta / p_{T}(\mu)<0.05$.

The vector boson scattering signature is exploited as well to further reduce the backgrounds contribution. The tag jets are identified as the ones with highest $p_{T}$ in the event. There will be very high fake rate for low $p_{T}$ jets, so the $p_{T}$ threshold of the tag jets is $30 \mathrm{GeV} / \mathrm{c}$. A number of different strategies to implement tag jets selection were compared, and the best rejection factor for a given efficiency is obtained by requiring the tag jets with the opposite sign of pseudo-rapidity $(\eta)$, to satisfy the $\eta$ difference $\Delta \eta_{j j}>4$ and tag jets invariant mass $m_{j j}>600 \mathrm{GeV} / \mathrm{c}^{2}$.

The event number after the cut-based selection for signal and background are shown in Table 1 . The results are normalized to an integrated luminosity of $60 \mathrm{fb}^{-1}$. For $t \bar{t}$, $\mathrm{W}+$ jet, single top and di-boson backgrounds, Monte Carlo samples corresponding to $60 \mathrm{fb}^{-1}$ are too large to be simulated, due to the very large cross section. Only few events survive after the selection chain with high statistics error.
Table 1 Number of surviving events for signal and background after muon and jet selection with an integrated luminosity of $60 \mathrm{fb}^{-1}$

\begin{tabular}{lll}
\hline$m_{\mathrm{H}}=200 \mathrm{GeV} / \mathrm{c}^{2}$ & no-Higgs & Backgrounds \\
\hline 12.2 & 13.7 & 5.9 \\
\hline
\end{tabular}

The expected number of events therefore will be estimated with the efficiency factorization as discussed below.

\section{Higgs versus no-Higgs scenario}

To distinguish the scenario where the Higgs boson is existing from the one where the Higgs boson is absent, two possible additional selections have been investigated. We choose the following relative separation definition to optimize the selections:

$\alpha=\frac{N_{\mathrm{NoH}}-N_{m_{\mathrm{H}}(200)}}{\sqrt{N_{m_{\mathrm{H}}(200)}+N_{\mathrm{Bkg}}}}$,

where $N_{m_{\mathrm{H}}(200)}, N_{\mathrm{NoH}}$ and $N_{\mathrm{Bkg}}$ are the number of events for the two cases and for the backgrounds respectively. For this study the value of the Higgs boson mass is not relevant, as explained in detailed in Ref. [7].

The region of high values of invariant mass of $\mathrm{W}$ bosons ( $m_{\mathrm{WW}}$ ) should be sensitive to the presence of a Higgs particle (Fig. 2). Unfortunately, because of the presence of neutrinos, it is impossible to reconstruct the invariant mass of the $\mathrm{W}$ bosons. Therefore, the invariant mass of the two muons system is used to replace $m_{\mathrm{WW}}$ and the events count for (4) is performed after a cut on the $m_{\mu \mu}$ value.

Figure 3(a) shows the $m_{\mu \mu}$ distribution for the two scenarios $\left(m_{\text {Higgs }}=200 \mathrm{GeV} / \mathrm{c}^{2}\right.$ and no-Higgs). Figure 3(b) shows the number of surviving signal events as a function of the $m_{\mu \mu}$ cut. Figure 3(c) shows the distribution of the relative separation (as defined in (4)) vs. the cut on $m_{\mu \mu}$. To obtain a better separation between the two cases, we require the muon to be in the central region: $\left|\eta_{\mu}\right|<2$. By asking $m_{\mu \mu}>200 \mathrm{GeV} / \mathrm{c}^{2}$, we can achieve good signal significance and background control. However, the request is too tight, since it eliminates about $80 \%$ of signal events (Fig. 3(b)).

Alternatively, a selection on the azimuthal angle between muons is investigated, as the vector bosons tend to be back to back in a scattering topology. Figure 4(a) shows the $\Delta \phi$ distribution between the two muons for the two cases. Figure 4(b) shows the number of surviving events as a function of a minimum $\Delta \phi_{\mu \mu}$ cut. Figure 4(c) is the distribution of the relative separation as defined in (4) vs. different $\Delta \phi_{\mu \mu}$ cuts. With the cut $\Delta \phi_{\mu \mu}>2$, the highest separation is obtained with a loss of about $50 \%$ of signal events. Only QCD and irreducible backgrounds are considered in Fig. 3(c) and Fig. 4(c). 


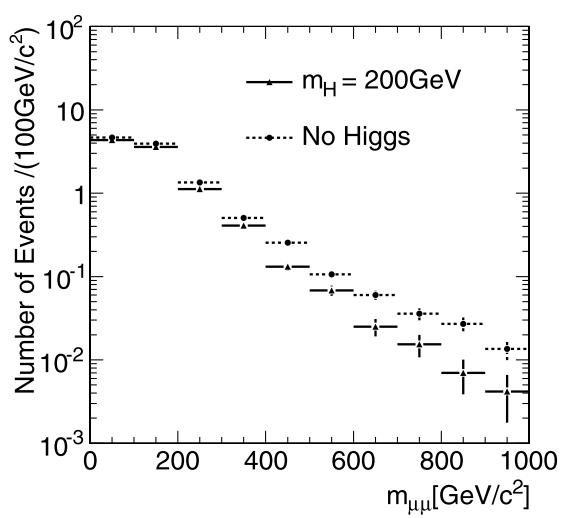

a

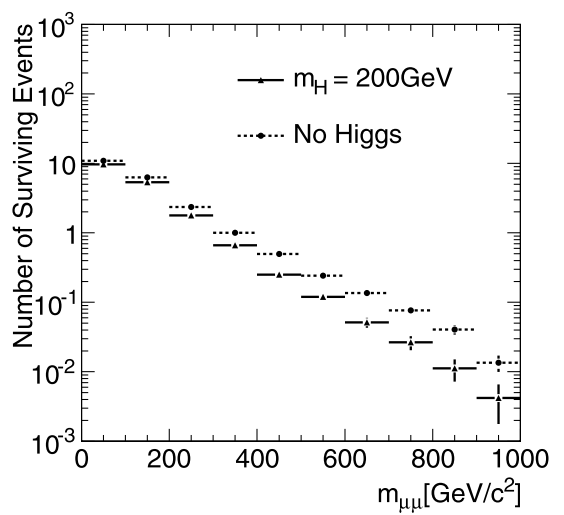

$\mathrm{b}$

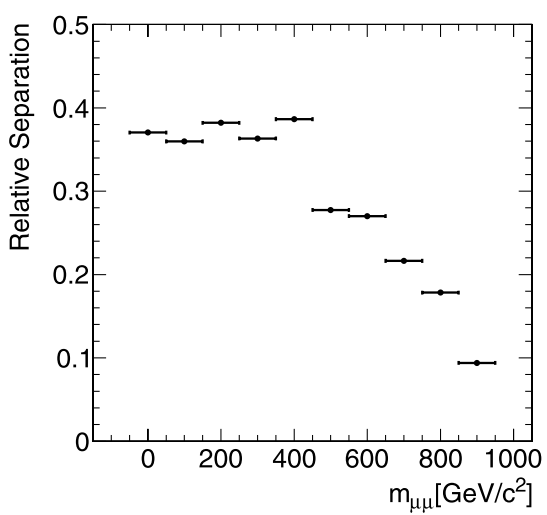

c

Fig. 3 Invariant mass distribution of the two muons $\left(m_{\mu \mu}\right)(\mathbf{a})$, the number of surviving events as a function of the cut on $m_{\mu \mu}(\mathbf{b})$, relative separation $\alpha$ vs. the $m_{\mu \mu}$ cut value (c). Results are normalized to $60 \mathrm{fb}^{-1}$

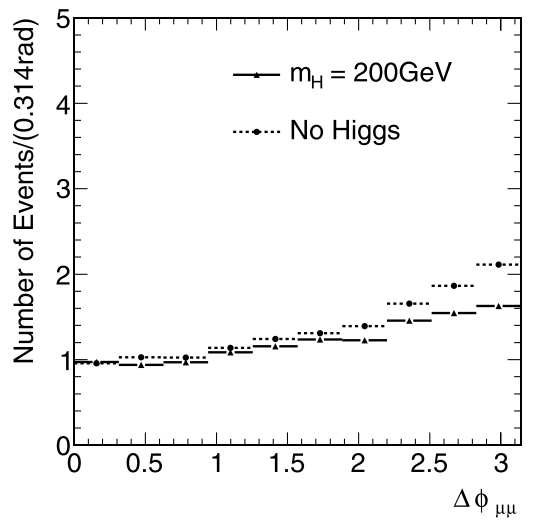

a

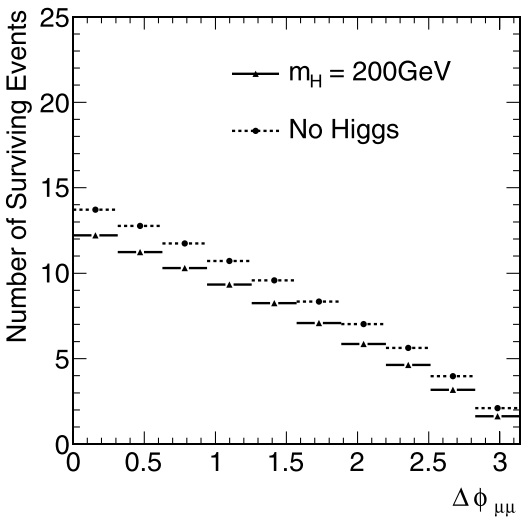

$\mathrm{b}$

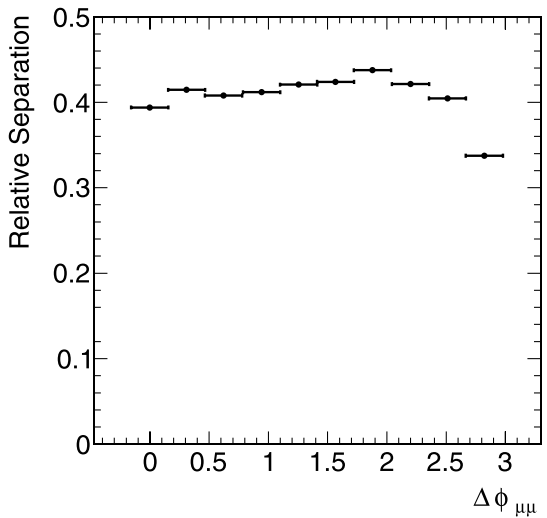

Fig. $4 \Delta \phi_{\mu \mu}$ distribution (a), the number of surviving events as a function of the $\Delta \phi_{\mu \mu}$ cut (b), relative separation $\alpha$ vs. the $\Delta \phi_{\mu \mu}$ cut value (c). Results are normalized to $60 \mathrm{fb}^{-1}$

\section{Background estimation}

The main uncertainty comes from the simulated background samples statistical error. Because of the limited statistics available, no event remains for $\mathrm{W}+$ jet, top and di-boson samples. However, we cannot ignore those backgrounds because of their very large cross sections.

Assuming there is no correlation among the single selections, we estimate the number of surviving events by multiplying the single efficiencies:

$N=\sigma \times L\left(60 \mathrm{fb}^{-1}\right) \times \xi_{\text {cut } 1} \times \xi_{\text {cut } 2} \cdots \times \xi_{\text {cut } i}$,

where the $\xi_{\text {cut } i}$ is the efficiency for the $i$-th selection alone on each sample. There is a very low level of correlation between the two main selections, namely the jet selections and muon selections. The expected number of background events for each sample using two different discriminators are summarized in Table 2 with an integrated luminosity of $60 \mathrm{fb}^{-1}$.
Table 2 Estimated number of events of backgrounds

\begin{tabular}{llll}
\hline Discriminator & Top & $\mathrm{W}+$ jet & di-boson \\
\hline$m_{\mu \mu}$ & 0.65 & 0.05 & 0.02 \\
$\Delta \phi_{\mu \mu}$ & 2.6 & 0.2 & 0.1 \\
\hline
\end{tabular}

The signal significance is determined using the likelihood ratio method, with poissonian probability density distributions, for both the $m_{\mu \mu}$ and $\Delta \phi_{\mu \mu}$ selections with the background estimates in Table 2. Results are listed in Table 3. The number of signal and background events are shown after the selection. We make the hypothesis that the correlation between the cuts will give $100 \%$ uncertainty for $\mathrm{W}+$ jet, top and di-boson backgrounds. For the other samples, only the statistical error is considered. 
Table 3 Signal significances, ratio and separation power between Higgs case and no-Higgs case with an integrated luminosity of $60 \mathrm{fb}^{-1}$, at $10 \mathrm{TeV}$ center-of-mass energy

\begin{tabular}{lllllll}
\hline Discriminator & $\mathrm{NoH}$ & $m_{\mathrm{H}}(200)$ & $\mathrm{NoH} / m_{\mathrm{H}}(200)$ & Background & Relative separation & $S_{\left(m_{\mathrm{H}}(200)\right)}$ \\
\hline$m_{\mu \mu}$ & $2.4 \pm 0.1$ & $1.8 \pm 0.1$ & $1.3 \pm 0.1$ & $1.0 \pm 0.8$ & 0.35 & 1.5 \\
$\Delta \phi_{\mu \mu}$ & $6.5 \pm 0.1$ & $5.4 \pm 0.1$ & $1.2 \pm 0.1$ & $3.5 \pm 2.9$ & 0.36 & 1.9 \\
\hline
\end{tabular}

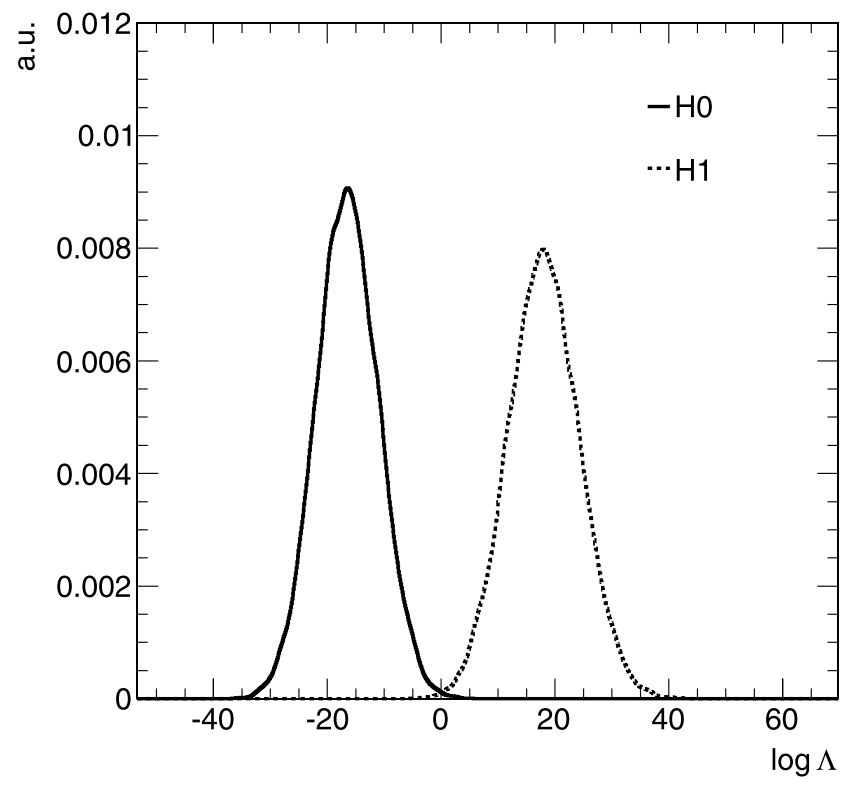

Fig. 5 Normalized Likelihood Ratio with $m_{\mu \mu}$ cut, H0 hypothesis is $m_{H}=200 \mathrm{GeV}, \mathrm{H} 1$ is no Higgs. Result is corresponding to an inverse luminosity of $6 \mathrm{ab}^{-1}$ at $\sqrt{s}=14 \mathrm{TeV}$

\section{Summary and Discussion}

Assuming a poissonian pdf of the measurements in the Higgs boson existing scenario and Higgs boson absence scenario, a likelihood-ratio is built to distinguish the two hypotheses, giving the number of measured events. To assess the separation power of the analysis, a set of toy-montecarlo experiments have been generated for each of the cases, and the distributions of the corresponding likelihood-ratios have been compared. To evaluate the separation between the two curves, the distance between their maxima, normalized to their sigma, is calculated (the sigma is taken as the halfwidth of the narrowest interval containing $68 \%$ of the distribution):

$\delta=\frac{\left|\max (L L R)_{H 0}-\max (L L R)_{H 1}\right|}{\sigma_{H 0} \oplus \sigma_{H 1}}$

where $H 0$ and $H 1$ represent the Higgs boson and no-Higgs boson hypotheses respectively. Figure 5 shows the distributions when we scale the results by the total expected statistics collected by ATLAS and CMS (corresponding to an inverse luminosity of $6 \mathrm{ab}^{-1}$ at $14 \mathrm{TeV}$ of LHC center-of- mass energy). A $4 \sigma$ separation for the two hypotheses can be achieved.

We present an exploratory study of the same sign W scattering process with $\mathrm{W}$ decay into $\mu \nu$ as probe of Higgs boson existence in pp collisions at $\sqrt{s}=10 \mathrm{TeV}$. All the standard model backgrounds are considered, with detector effects parameterized, including muon mis-identification effect. It is a clean channel compared with the other VV scattering processes $[7,15]$ because of the two main signatures, which are the same sign isolated muons pair and energetic forward jets. $m_{\mu \mu}$ and $\Delta \phi_{\mu \mu}$ are both good discriminants to distinguish a Higgs scenario from the no-Higgs one.

Although the cross section is not as large as searching for Higgs Boson via di-boson resonances directly, it is a model independent channel to determine if the Higgs boson exists, whatever the value of its mass, and to verify the unitarity of the theory.

With the total statistics expected from the ATLAS and CMS experiments at $14 \mathrm{TeV}$, the separation power between Higgs boson and no-Higgs boson scenarios will be at the level of $4 \sigma$.

Open Access This article is distributed under the terms of the Creative Commons Attribution Noncommercial License which permits any noncommercial use, distribution, and reproduction in any medium, provided the original author(s) and source are credited.

\section{References}

1. M.J.G. Veltman, CERN-97-05

2. M.S. Chanowitz, hep-ph/9812215

3. S. Dawson, hep-ph/9901280

4. C. Quigg, Acta Phys. Pol. B 30, 2145 (1999). hep-ph/9905369

5. S. Dawson, Int. J. Mod. Phys. A 21, 1629 (2006). hep-ph/0510385

6. R. Rattazzi, PoS HEP 2005, 399 (2006). hep-ph/0607058

7. E. Accomando et al., J. High Energy Phys. 0603, 093 (2006)

8. J. Bagger et al., Phys. Rev. D 52, 3878-3889 (1995)

9. A. Ballestrero, A. Belhouari, G. Bevilacqua, et al., Comput. Phys. Commun. 180, 401-417 (2009)

10. The CMS Collaboration, CERN-LHCC-2006-001

11. F. Maltoni, T. Stelzer, J. High Energy Phys. 0302, 027 (2003). arXiv:hep-ph/0208156

12. T. Stelzer, W.F. Long, Comput. Phys. Commun. 81, 357 (1994). arXiv:hep-ph/9401258

13. T. Sjostrand, S. Mrenna, P. Skands, J. High Energy Phys. 0605, 026 (2006)

14. The ATLAS Collaboration, CERN-LHCC-1999-14, vol. 1

15. A. Sznajder (for CMS Collaboration), arXiv:0810.3604v2 